\title{
Emergency airway management of tracheo-innominate fistulas in the pediatric population
}

\author{
Jay B. Tuchman, MD • M. Concetta Lupa, MD • \\ Edmund H. Jooste, MB, ChB
}

Received: 17 February 2010/Accepted: 18 May 2010/Published online: 5 June 2010

(c) Canadian Anesthesiologists' Society 2010

Tracheo-innominate fistula (TIF) is a rare but well recognized complication of tracheostomy placement, and it is universally fatal in the absence of surgical management. Adult algorithms for TIF management do not address the unique dilemmas that exist in the pediatric population with respect to uncuffed tracheostomy tubes (TT). We present a case that highlights the need to modify the adult algorithm when applying it in children. Parental informed consent has been obtained for publication of the following case.

A seven-year-old $25 \mathrm{~kg}$ encephalopathic ventilatordependent patient with a 4.0 uncuffed TT presented with emergency tracheostomy bleeding, which resulted in an inability to ventilate and subsequent loss of airway. The attending anesthesiologist was summoned immediately to the patient's bedside and elected to secure the airway by placing a 4.0 cuffed endotracheal tube (ETT) through the tracheostomy stoma. Profuse bleeding ensued within an hour, and the patient was transported immediately to the operating room for further management.

The patient was tachycardic and hypertensive, with 95\% oxygen saturation on $100 \% \mathrm{O}_{2}$ and unobtainable end-tidal $\mathrm{CO}_{2}$ due to copious blood in the sampling line. An earnose-and-throat (ENT) surgeon performed rigid bronchoscopy, confirming the diagnosis of TIF, and partially blocked the bleeding fistula by applying anterior pressure against the sternum. With hyperinflation of the cuff of the ETT and application of direct pressure with the rigid bronchoscope, the bleeding decreased so central venous

J. B. Tuchman, MD $(\bowtie) \cdot M$. Concetta Lupa, MD .

E. H. Jooste, MB, ChB

Children's Hospital of Pittsburgh, Pittsburgh, PA, USA

e-mail: tuchmanj@upmc.edu and arterial accesses could be secured. A sternotomy was then performed, allowing identification and resection of a $0.5 \mathrm{~cm}$ TIF. Postoperatively, the ETT was sutured in place via the tracheostomy stoma and replaced by an uncuffed TT on postoperative day nine. The patient was discharged home at baseline condition a few days later.

Previous reports have typically described the existing adult management algorithm being completely appropriate in pediatric patients with a cuffed TT who were older than age ten. ${ }^{1,2}$ The adult algorithm involves TT cuff hyperinflation distal to the fistula. If hyperinflation proves unsuccessful, digital compression of the innominate artery against the sternum via the tracheostomy stoma should be attempted. $^{3}$ This maneuver has been reported as being successful in up to $90 \%$ of cases. ${ }^{4}$ It is important to recognize that the paucity of prior pediatric reports may be attributed to the primary utilization of uncuffed TT and the rarity of TIF in the pediatric population younger than age eight. ${ }^{5}$ This is highlighted by the longstanding recommendation in major pediatric textbooks to place an uncuffed ETT in all patients younger than age eight. With increased cuffed TT utilization, one might posit that this pediatric complication may become more prevalent in the future. Therefore, despite the current rarity of pediatric TIF in patients with an uncuffed TT, we feel that this alternate algorithm may be critical to the survival of future patients.

There are several major distinctions between the adult algorithm and our proposed algorithm for emergency management of pediatric patients with uncuffed trachestomies (Figure). Cuff hyperinflation, recommended as the first step in most adult algorithms, is an obvious impossibility in most pediatric patients with an uncuffed TT. Additionally, the small pediatric airway does not allow for the next step of digital compression through the stoma, as 
Figure Arterial bleeding from tracheostomy tube or site

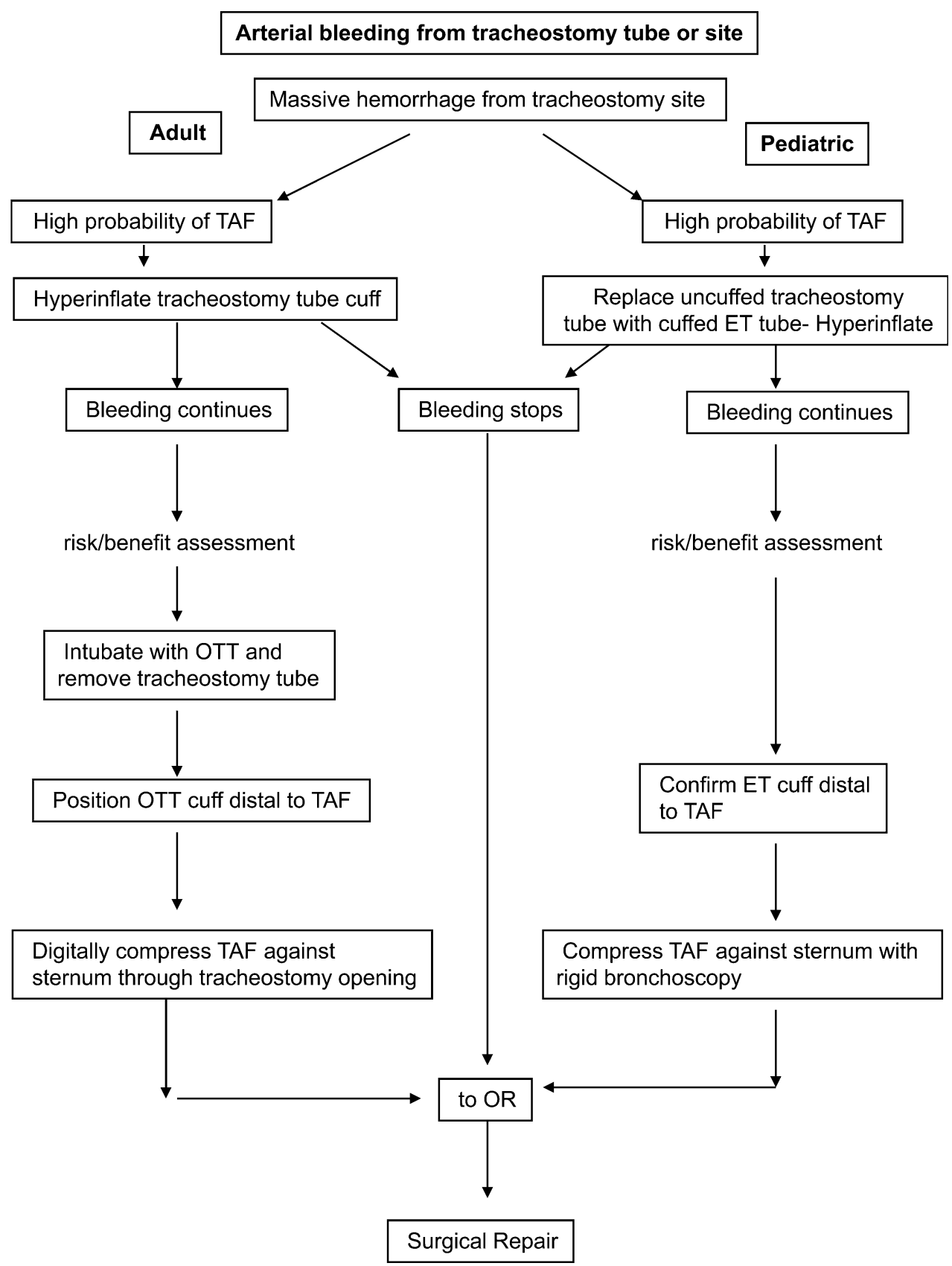

this may lead to significant airway obstruction. Specifically, in the small airway of our patient, the maneuvers suggested by the adult algorithm simply were not feasible. Instead, our pediatric algorithm suggests replacement of the uncuffed TT with a hyperinflated cuffed ETT via the stoma, positioned distal to the fistula. Rigid bronchoscopy by an ENT surgeon should follow, with the dual purpose of diagnostic visualization, and more importantly, fistula compression, allowing critical preparation time for the definitive cardiothoracic surgical procedure.

Maintaining a high index of suspicion, communicating between specialists, and following a clearly defined management algorithm may greatly facilitate timely and proficient management crucial for survival.

\section{References}

1. Ghai B, Makkar JK, Bakshi J, Rana S, Singh RS, Bhagat S. Survival of a child without sequelae after tracheoarterial fistula. Paediatr Anaesth 2007; 17: 588-91.

2. Joshi AS, Tanna N, Elmaraghy CA, et al. Nonsurgical treatment of tracheoinnominate fistula in the pediatric population. Arch Otolaryngol Head Neck Surg 2007; 133: 294-6. 
3. Kapural L, Sprung J, Gluncic I, et al. Tracheo-innominate artery fistula after tracheostomy. Anesth Analg 1999; 88: 777-80.

4. Jones JW, Reynolds M, Hewitt RL, Drapanas T. Tracheoinnominate artery erosion: successful surgical management of a devastating complication. Ann Surg 1976; 184: 194-204.
5. Johnson PE, Tabaee A, Fitz-James IA, Pass RH, de Serres LM. Major aorto-pulmonary collateral arteries (MAPCAs)-Bronchial fistula presenting as tracheotomy bleed. Int J Pediatr Otorhinolaryngol 2006; 70: 1109-13. 